\title{
Nature of Copper Active Sites in CuZSM-5: Theory and Experiment
}

\author{
Ewa Broclawik $^{1 *}$ Jerzy Datka ${ }^{2}$, Barbara Gil $^{2}$ and Pawel Kozyra ${ }^{2}$ \\ ${ }^{1}$ Institute of Catalysis, Polish Academy of Sciences, ul. Niezapominajek 8, 30-239 Cracow, Poland. \\ E-mail:broclawi@chemia.uj.edu.pl \\ ${ }^{2}$ Faculty of Chemistry, Jagiellonian University, ul. Ingardena 3, 30-060 Cracow, Poland \\ *Author to whom correspondence should be addressed.
}

Received: 11 October 2001 / Accepted: 31 January 2002 / Published: 25 April 2002

\begin{abstract}
We report here a concise resume reporting the way of constructing the model of an active site composed of transition metal cation exchanged in zeolites. The main goal was to devise the model of CuZSM-5 capable of describing geometrical and electronic properties of metal sites and adsorption complexes with small molecules. The models were built up starting from simple ring structures encountered in ZSM-5 framework to fused rings' model selected as the representative of $\alpha$ position for hosting the exchanged cation. Geometrical and electronic properties of the basal model, composed of the extended framework cluster with $\mathrm{Cu}^{+}$or $\mathrm{Cu}^{2+}$ cation, and adsorption complexes with diatomic molecules were extracted from DFT calculations. The stress was put here on direct confirmation of structural changes on copper reduction/oxidation and adsorption. Electron donor/acceptor properties of the sites combined with electronic properties of adsorbed molecules led to the proposal for the mechanism of $\mathrm{NO}$ activation by $\mathrm{Cu}^{+} \mathrm{ZSM}-5$ : transfer of electrons from copper d orbitals to antibonding states of NO should cause large weakening of the bond, which was evidenced also by IR measurements.
\end{abstract}

Keywords: CuZSM-5, deNOx, active sites, molecular modeling, density functional theory.

\section{Introduction}

The question about the source of a very high activity of metal sites produced by cation exchange in a silicalite environment of framework oxygens is the important area of the research in zeolite 
community. One of the main points of interest is the $\mathrm{Cu}^{+}$centre in $\mathrm{Cu}^{+} \mathrm{ZSM}-5$, known as a good catalyst for decomposition of nitrogen oxides [1], classified among the most dangerous pollutants. Another interesting property of $\mathrm{Cu}^{+}$in ZSM-5 is the possibility of bonding $\mathrm{N}_{2}$ at room temperature [2]. In spite of numerous efforts, however, little is still known about the nature of the $\mathrm{Cu}^{+}$site. Experimental difficulties (e.g. in XRD) stem from low content of framework aluminum and hence very low concentration of copper sites.

$\mathrm{Cu}^{+}$sites are produced from $\mathrm{Cu}^{2+}$ exchanged cations by the process of self-reduction during dehydration and a number of hypotheses have been proposed as to the structure and coordination of both copper forms $[3,4]$. In addition, the interaction with NO molecule also triggers out reductionoxidation processes leading to the modification of the site structure and properties. Experimental techniques can reach only selected properties: while $\mathrm{Cu}^{2+}$ is EPR visible centre, the reduced form cannot be followed by this tool; on the contrary, the complex with NO is EPR silent for the $\mathrm{Cu}^{2+}$ form. $\mathrm{XRD}$ which is useful for $\mathrm{Al}$ rich zeolites has several limitations in the case of high siliceous zeolites because of low cation content and low symmetry of zeolite framework [5]. Nevertheless, the consensus has already been met that there exist several positions of $\mathrm{Cu}$ cation depending on the oxidation state and the interaction with an adsorbate [6-8]. The most probable coordination for $\mathrm{Cu}^{2+}$ is the fourfold bonded, approximately planar square structure, while $\mathrm{Cu}^{+}$prefers lower coordination between 2 and 3 exhibiting lower symmetry. $\mathrm{Cu}^{+}$are supposed to be compensated by a single framework aluminum atom while two framework aluminums are required to compensate a bare $\mathrm{Cu}^{2+}[9]$. In consequence many prospective sites hosting exchanged Cu cations in ZSM-5 framework have been proposed. It has already been suggested, e.g. from photoluminescence studies that among many possibilities accessible for $\mathrm{Cu}^{+}$in ZSM-5 two positions: $\alpha$ and $\beta$ are prevailing while one of them $(\alpha)$ is suggested to be catalytically active [10]. In view of the limited experimental information available on $\mathrm{Cu}$ binding sites molecular modelling of their properties is burdened with high degree of arbitrariness and uncertainty and always needs additional support and justification by parallel experimental measurements.

IR spectroscopy provides another insight into the nature of copper sites in ZSM-5: it is the best probe of modifications undergone by the adsorbed molecule and, indirectly, gives some information on the influence of the presence of exchanged cation and the adsorbed molecule onto the site structure and framework properties. As already known, such information may be extracted both from the analysis of NO stretch and of T-O-T skeletal vibration [11-15]. In this paper, on the basis of our theoretical investigations related to experimentally available information, we discuss the problem of speciation of $\mathrm{Cu}$ ions in MFI framework and its migration in the course of a catalytic process.

\section{Methodological remarks}

Nowadays, molecular modelling emerged as the new, complementary tool to study properties of active sites in catalysis. In view of incompleteness of experimentally available information on cationexchanged ZSM-5 and the need to devise a model of such site, which would be helpful in validation of 
working hypotheses, we have undertaken quantum chemical calculations for the part of MFI framework capable of hosting copper cations. We have already tested several small cluster models built of $6 \mathrm{~T}$ or $5 \mathrm{~T}$ rings cut-off the MFI framework as prospective hosts for $\mathrm{Cu}^{2+}$ and $\mathrm{Cu}^{+}[15,16]$. These models were capable of describing geometrical properties of the site, which was confirmed by very good correlation between calculated framework distortion imposed by the cation and shifts in TO-T skeletal vibration measured by IR spectroscopy [15]. We have found that $\mathrm{Cu}^{2+}$ prefers fourfold coordination in the centre of six-ring while $\mathrm{Cu}^{+}$becomes bonded to three bridging oxygens in a $5 \mathrm{~T}$ ring. It was also possible to partly assess properties of adsorbed small diatomic molecules and thus activating ability of the site [16]. However, these models appeared to be too small to fully account for geometry modifications caused by the strong interaction with the sorbed molecule, in particular NO. In small 5T or 6T models each form of the copper cation, $\mathrm{Cu}^{2+}$ and $\mathrm{Cu}^{+}$, had to be described by a different cluster since $\mathrm{Cu}^{+}$was not bound in the centre of $6 \mathrm{~T}$ ring. Thus quantitative comparison of their properties could not be performed, even if their qualitative comparison was sufficiently convincing. In addition, the interaction with the sorbed NO molecule appeared to be so strong that the copper became withdrawn from the centre of small 6T or 5T models and became shifted to cluster peripheries. Thus the adsorption complex could not be fully described and direct vibrational analysis would not be properly justified. The effect could be partly due to unrealistic rigity of such small, constrained models. In bigger models the influence of freezing terminal hydrogens could be expected less significant, in accordance with other experience.

Thus, in view of our final goal which was to study modifications of the site structure and properties imposed by reduction-oxidation and adsorption processes, it was indispensable to devise the model capable of simultaneously describing a variety of copper forms and their adsorption complexes with NO. For this purpose extended models should have been considered, which would include larger parts of the framework indispensable to distinguish between structural fragments, large enough to be less sensitive to saturation scheme. Such approach has already been pursued by many authors, within both semiclassical [17] and more rigorous quantum chemical regime [18-21]. On the basis of previous experience of other researchers [20,21] and our own studies the following model has been proposed for the $\alpha$ position in ZSM-5 after careful probing several possibilities. The cluster composed of two fused $5 \mathrm{~T}$ rings forming simultaneously the $6 \mathrm{~T}$ ring, with two aluminums positioned at the five-ring junctions is shown schematically in Fig. 1. We call this 7T cluster model the basket model. We did not attempt here to test variety of sites and models as it has already been done by others (see e.g 20,21). We rather tried to follow one site and model, recommended by existing theoretical and experimental studies and extend the study with direct analysis of NO sorption and activation properties. Cluster boundaries were saturated by $\mathrm{OH}^{-}$groups, which gave neutral cluster models according to commonly accepted saturation scheme. During geometry optimisation the positions of terminating hydrogens have been fixed to keep the parent framework structural features. There exist many approaches to mimic missing bulk of the solid, including variety of saturation and embedding schemes. We have followed relatively 
simple one in hope that when the model becomes larger the saturation scheme may become less important. The advantage of this approach was easy access to molecular properties including frequencies from full vibrational analysis.

Quantum chemical calculations for this system were performed with the use of modern implementation of DFT methodology, acknowledged for its high accuracy and good performance for transition metal systems (DMol of Molecular Simulations, Inc.[22]). There are two types of DFTbased codes. In non-HF based schemes like DMol being pure numerical implementation of KohnSham equations, LDA approach although approximate, is the one with best understood physical background and was frequently shown to lead to reasonable geometries and surprisingly good frequencies. This is not so clear with Hartree-Fock based codes where more sophisticated exchangecorrelation potentials like three-parameter B3LYP are rather obligatory. Thus standard calculational parameters e.g. local VWN potential and DNP basis set were used in this work since it has already been confirmed in our previous studies for transition metal systems that such choice leads to good geometries, vibrational frequencies and qualitatively acceptable energetic parameters [23]. The calculations provided minimum energy structures after constrained geometry optimisation, the character of the wave function giving electronic structure of the sites and charge distribution. Charge distribution was obtained from Hirshfeld population analysis, advised for numerical DFT calculations. For the systems with adsorbed NO molecule full vibrational analysis was performed providing frequencies to be compared with infrared measurements. We did not use any scaling factor as it was frequently shown to be not necessary for DFT calculations, even within LDA approximation. We were aware that the diagonalisation of the Hessian matrix for geometry obtained from constrained minimisation could be not fully justified thus we have checked carefully the nature of normal modes connected to the stretch of NO molecule. We have found the NO stretching frequency to be very well resolved from other vibrations and not contaminated.

\section{Results}

Fig. 1 shows the optimised positions of $\mathrm{Cu}^{2+}$ and $\mathrm{Cu}^{+}$in the common basket model of the $\alpha$ site in ZSM-5. Column 2 in Table 1 gives the distances between the appropriate cation and nearest framework oxygens (below $2.5 \AA$ ). It can be easily verified that divalent copper prefers planar square coordination in the centre of $6 \mathrm{~T}$ ring of the $7 \mathrm{~T}$ model while monovalent copper shifts towards the centre of the $5 \mathrm{~T}$ part of the model forming three bonds with bridging Al-O-Si oxygens in a five-ring. Nevertheless, both forms of the cation remain bonded in the central area of the model. The shift between the positions of $\mathrm{Cu}^{2+}$ and $\mathrm{Cu}^{+}$sites in our model amounts to $1.22 \AA$. This result directly confirms the assumption of different environment for both forms of copper cation and its migration in the zeolite framework upon reduction-oxidation. The position of monovalent copper differs significantly from the one obtained by Nachtigallova et al. [21], where the difference between the positions of $\mathrm{Cu}^{2+}$ and $\mathrm{Cu}^{+}$was not so distinct. Thus, we have recalculated this model and we have 


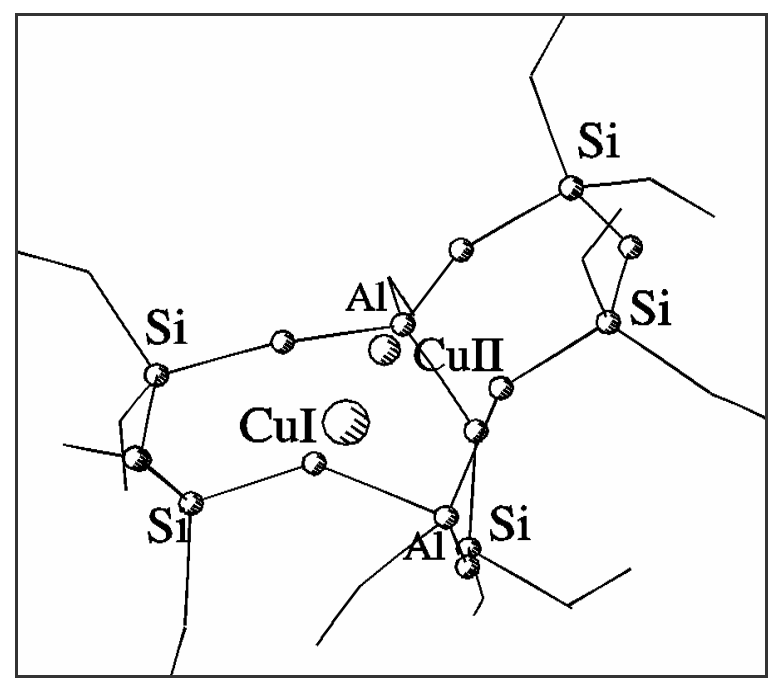

Figure 1. Structure and coordination of $\mathrm{Cu}^{2+}$ and $\mathrm{Cu}^{+}$sites in a common $7 \mathrm{~T}$ basket

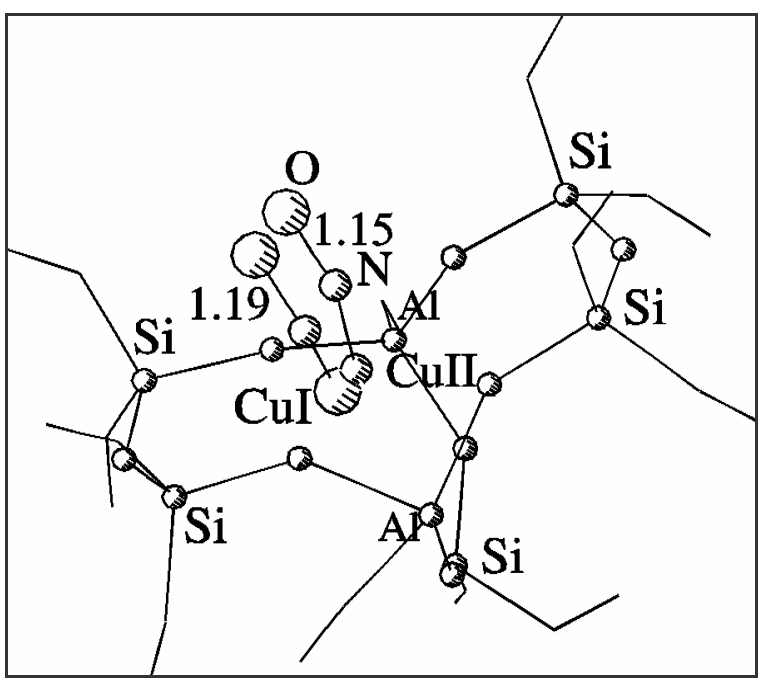

Figure 2. Geometry of $\mathrm{NO}$ adsorption on $\mathrm{Cu}^{2+}$ and $\mathrm{Cu}^{+}$sites in a common $7 \mathrm{~T}$ basket model of CuZSM-5

found the other geometry to be the local minimum slightly higher in energy. In addition, geometrical parameters of both sites agree perfectly with those obtained previously for smaller, single-ring models $[15,16]$. Therefore we regard the $7 \mathrm{~T}$ basket model as the smooth extension of our previous single-ring models.

Thus in the next step the extended 7T basket model was used to study properties of adsorption complexes of nitrogen oxide with exchanged copper cations. Fig. 2 shows superimposed optimised geometries of $\mathrm{NO}$ adduct with $\mathrm{Cu}^{2+}$ and $\mathrm{Cu}^{+}$sites in ZSM-5. The NO molecule is strongly bonded by both $\mathrm{Cu}^{2+}$ and $\mathrm{Cu}^{+}$sites. NO adsorption modifies the position of the cation and its interaction with the framework, which is particularly effective for $\mathrm{Cu}^{2+}$. Column 3 in Table 1 lists the distances between the appropriate cation interacting with nitrogen oxide and nearest framework oxygens. The cation becomes withdrawn by the interaction from its original position and changes its coordination to the threefold one close to the centre of the 5T part of the model. The interaction of $\mathrm{Cu}^{+}$with $\mathrm{NO}$ is weaker and the modification of its coordination is much smaller than that of $\mathrm{Cu}^{2+}$, although the shift of $\mathrm{Cu}^{+}$to $\mathrm{Cu}^{2+}$ position is also observed. The distance between the positions of the two copper cations decreases from $1.22 \AA$ to $0.36 \AA$ when interacting with NO, which indicates additional copper migration after

Table 1. Bond distances between copper cations and bridging oxygens below $2.5 \AA$, (index ${ }_{5 \mathrm{~T}}$ denotes oxygen in $5 \mathrm{~T}$ ring, otherwise in $6 \mathrm{~T}$ ring).

\begin{tabular}{ccc}
\hline & \multicolumn{2}{c}{$\mathrm{R}_{\mathrm{Cu}-\mathrm{O}}[\AA]$} \\
\cline { 2 - 3 } Centre in M7 & Site & Site with NO \\
\hline $\mathrm{Cu}^{2+}$ & $2.03,2.03,2.05,2.05$ & $2.02,2.03,2.10_{5 \mathrm{~T}}$ \\
$\mathrm{Cu}^{+}$ & $1.93,2.01,2.03_{5 \mathrm{~T}}$ & $2.02,2.06,2.06_{5 \mathrm{~T}}$
\end{tabular}


adsorption. These detailed properties of the sites and their adsorption complexes with NO could not be obtained with the use of simplified single-ring models even if they were verified to provide good description of selected geometrical properties of the sites.

The two preceding paragraphs showed that very bride scope of valuable information could be extracted from the proposed extended model. On the other side, the model was by no means perfect and burdened with high degree of arbitrariness similarly to all molecular models of extended systems. Thus it was highly desirable to confirm its validity by testing against existing experiment in the similar manner to that utilised for the first time in the case of small models [15]. There we used the mean value of deviation between cartesian coordinates of superimposed structures with and without guest moiety $(\mathrm{Cu}$ or $\mathrm{NO})$ as the measure of framework distortion after cation exchange or NO sorption and correlated this parameter with known shifts in skeletal vibration. This seemed to be the only way of theoretical interpretation of such shifts because skeletal vibration frequencies could not be obtained from direct vibrational analysis since this is physically extended motion and could be seriously perturbed by cluster model calculations and contamination with artificial boundary conditions. Here the basket model has been again validated via indirect interpretation of shifts in T-O-T skeletal frequencies caused by cation exchange and NO sorption, measured by infrared spectroscopy [11-15]. Calculated parameters characterising geometrical framework distortion were very similar to those determined for small models and changed consequently with the shift of skeletal vibration. In this work we propose additional way to approach the problem of interpreting shifts in skeletal vibration. TO-T vibration may be viewed as a combination of T-O stretching frequencies and thus its shift should correlate with the average strength of T-O bonds in the vicinity of perturbing cation. In the M7 model the positions of both forms of copper cation and their complexes with NO could be precisely determined and therefore we could additionally correlate shifts in T-O-T frequency with calculated changes of Mayer bond orders of T-O pairs for all Al-O-Si bridges in the model.

Inspection of Table 2 shows that red shift in T-O-T frequency corresponds perfectly to global weakening of these $\mathrm{T}-\mathrm{O}$ bonds, where the effect of $\mathrm{Cu}^{2+}$ is the strongest. Table 2 shows also that the interaction with NO substantially weakens the $\mathrm{Cu}^{2+}-\mathrm{MFI}$ framework interaction and makes the T-O bonds stronger what makes the perturbing effect of $\mathrm{Cu}^{2+}$ on the framework comparable to that of $\mathrm{Cu}^{+}$. It is consistent with previous discussion of geometrical changes caused by adsorption where we have already shown that $\mathrm{Cu}^{2+}$ cation is withdrawn by the interaction with NO from its original position and changes its coordination to the threefold one. The interaction of $\mathrm{Cu}^{+}$with $\mathrm{NO}$ is weaker and the modification of its coordination is much smaller than that of $\mathrm{Cu}^{2+}$, although the shift of $\mathrm{Cu}^{+}$towards $\mathrm{Cu}^{2+}$ position is also observed. In this way predictions concerning the nature of a cation-framework and cation-sorbed molecule interaction, inferred from geometrical analysis of the models, acquires experimental support. 
Table 2. The frequency shift from the position of unperturbed ring $\left(\Delta v=1020 \mathrm{~cm}^{-1}-v\right)$ of IR band of T$\mathrm{O}-\mathrm{T}$ vibration for $\mathrm{Cu}^{2+}$ and $\mathrm{Cu}^{+}$ions and their complexes with $\mathrm{NO}[11,14]$, and calculated changes in sums of T-O bond orders.

\begin{tabular}{ccccc}
\hline & $\mathrm{Cu}^{2+} \mathrm{ZSM}-5$ & $\mathrm{Cu}^{+} \mathrm{ZSM}-5$ & $\mathrm{Cu}^{2+}-\mathrm{NO}$ & $\mathrm{Cu}^{+}-\mathrm{NO}$ \\
\cline { 2 - 5 }$\Delta \mathrm{v} \mathrm{cm}^{-1}$ & 83 & 43 & 58 & $\mathrm{a})$ \\
$\Delta(\text { (2b.o. })_{\mathrm{T}-\mathrm{O}}$ & -0.68 & -0.43 & -0.43 & -0.40 \\
\hline
\end{tabular}

a) cannot be measured (see ref. 15)

Table 3 displays the properties of $\mathrm{Cu}-\mathrm{NO}$ adsorption complexes obtained from electronic structure calculations for the basket model. Indeed, the adsorption energy of nitrogen oxide on $\mathrm{Cu}^{2+} \mathrm{ZSM}-5$ is much higher than that on $\mathrm{Cu}^{+} \mathrm{ZSM}-5$ even if the bond length and bond angle is similar in each case. Distinct difference, however, shows up in the N-O bond distance. It becomes strongly elongated after sorption of $\mathrm{NO}$ on $\mathrm{Cu}^{+} \mathrm{ZSM}-5$ while the influence of $\mathrm{Cu}^{2+}$ site leads to slight shortening of the bond. This indicates that the nature of NO bonding with the active site in the two cases should be different. In order to discuss more precisely the features of the substrate - active site bonding additional electronic factors should be considered.

Table 3. Calculated properties of adsorption complexes between NO molecule and CuZSM-5: adsorption energy, equilibrium $\mathrm{Cu}-\mathrm{NO}$ bond distance and angle, intramolecular NO bond length.

\begin{tabular}{lcccc}
\hline Centre in M7 & $\begin{array}{c}\mathrm{E}_{\mathrm{ads}} \\
\mathrm{kcal} / \mathrm{mol}\end{array}$ & $\begin{array}{c}\mathrm{R}_{\mathrm{Cu}-\mathrm{NO}} \\
\AA\end{array}$ & $\begin{array}{c}\mathrm{Cu}-\mathrm{N}-\mathrm{O} \text { angle } \\
(\mathrm{deg})\end{array}$ & $\begin{array}{c}\mathrm{R}_{\mathrm{NO}} \\
\AA\end{array}$ \\
\hline $\mathrm{Cu}^{2+}-\mathrm{NO}$ & 40 & 1.80 & 128 & 1.15 \\
$\mathrm{Cu}^{+}-\mathrm{NO}$ & 24 & 1.84 & 132 & 1.19 \\
\hline
\end{tabular}

Table 4 lists parameters of the charge distribution and NO stretching frequencies in addition to simple geometrical factors. It is clearly visible that divalent copper withdraws electrons from the sorbed molecule and, in consequence, intramolecular NO bond becomes stronger (shorter by $0.01 \AA$ ). whereas monovalent copper donates electrons to the molecule and weakens the intramolecular bond, which becomes by $0.03 \AA$ longer. More critical inspection of the charge redistribution indicates, however, that the appropriate copper centre itself cannot be treated as the sole element of the active site. The calculations show that $\mathrm{Cu}^{2+}$ centre acquires only 0.07 e while NO looses $0.25 \mathrm{e}$, which must be redistributed over the framework serving as an electron reservoir Accordingly, $\mathrm{Cu}^{+}$looses 0.08 e on interaction, only partly on behalf of $\mathrm{NO}$ and the remainder must be again redistributed over the framework. Thus the MFI framework acts as a generalised soft ligand which serves as the buffer for electron redistribution processes and strongly influences the properties of the active site. 
Table 4. Effect of NO interaction with CuZSM-5: charge on copper cation and adsorbed NO, changes in $\mathrm{NO}$ bond lengths $\left(\Delta \mathrm{R}_{\mathrm{NO}}\right)$, calculated and measured NO stretching frequency $\left(v_{\mathrm{NO}}\right)$.

\begin{tabular}{lccccc}
\hline & $\mathrm{Q}_{\mathrm{Cu}}$ & $\mathrm{Q}_{\mathrm{NO}}$ & $\begin{array}{c}\Delta \mathrm{R}_{\mathrm{NO}} \\
\AA\end{array}$ & $\begin{array}{c}v_{\mathrm{NO}}(\mathrm{DFT}) \\
\mathrm{cm}^{-1}\end{array}$ & $\begin{array}{c}v_{\mathrm{NO}}(\mathrm{IR}) \\
\mathrm{cm}^{-1}\end{array}$ \\
\cline { 2 - 6 } $\mathrm{Cu}^{2+}-\mathrm{NO}$ & +0.43 & +0.25 & -0.01 & 1904 & $1895^{\mathrm{a})}$ \\
$\mathrm{Cu}^{+}-\mathrm{NO}$ & +0.36 & -0.03 & +0.03 & 1754 & $1809^{\mathrm{b})}$ \\
\hline
\end{tabular}

a) taken from refs. 24, 25

b) taken from ref. 16

Scheme 1 shows the energetic diagram for the highest occupied orbitals in the isolated subsystems calculated by DFT. To enrich the discussion also bare copper cations are included in the scheme. Inspection of the diagram allows for sketching the interpretation of electron phenomena occurring in the catalytic system composed of copper site in ZSM-5 and the NO molecule. Taking the position of the highest occupied level in the subsystem as the crude approximation of its Fermi level we can conclude that for bare copper cations the only process expected on interaction would be complete transfer of electrons from the molecule to the cation. Copper ligation by bridging oxygens from the MFI framework leads to significant shift of the Fermi level upwards which diminishes copper tendency to accept electrons and triggers out the increase of its donor properties. In the case of $\mathrm{Cu}^{+} \mathrm{ZSM}-5$ Fermi level approaches very closely the energy of semioccupied orbital of NO which enables electron backdonation. As the character of the highest occupied orbital of NO is strongly antibonding donation of electrons must lead to strong destabilisation of NO bond and, in consequence, to the activation of the molecule.

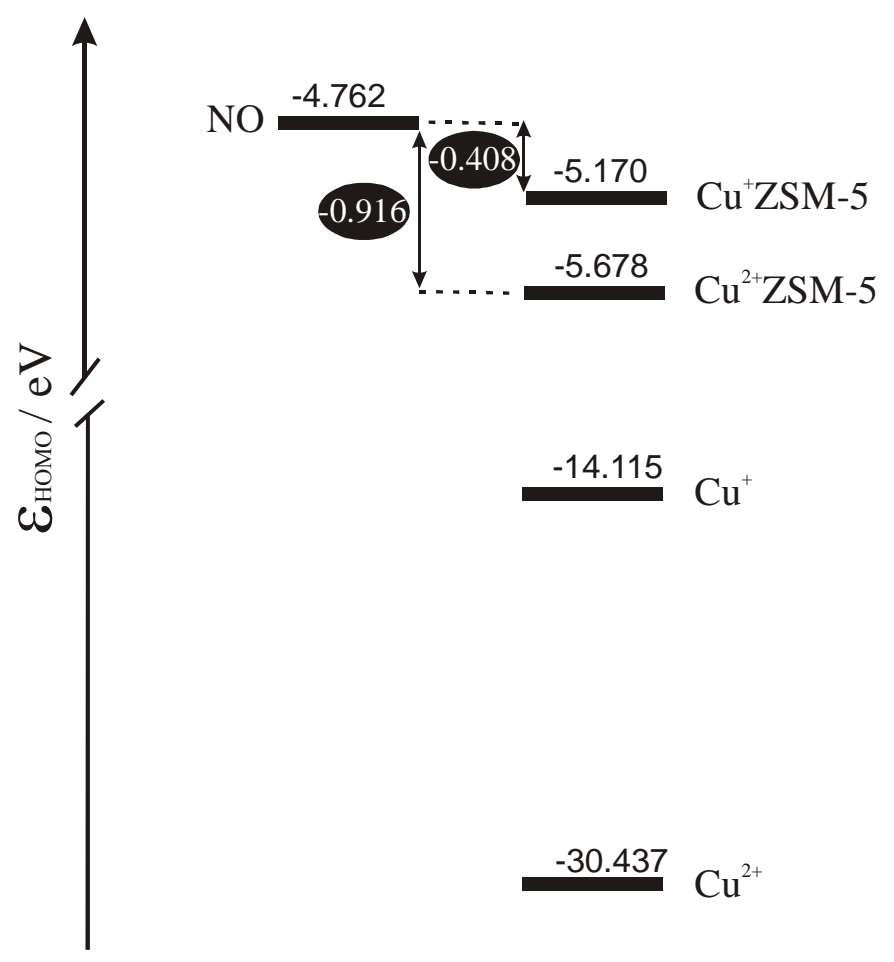

Scheme 1 
The same effects show in the NO stretching frequency, which is a good experimental measure of the bond strength. The values of $v_{\mathrm{NO}}$ measured by others [24,25] and by us [16] in IR spectroscopy, and calculated from our basket model [26,27] are listed in columns 5 and 6 in Table 4. Measured and calculated by us NO stretching frequency is equal to $1876 \mathrm{~cm}^{-1}$ and $1912 \mathrm{~cm}^{-1}$, respectively [26, 27]. In IR experiment blue shift of $v_{\mathrm{NO}}$ is observed for $\mathrm{Cu}^{2+} \mathrm{ZSM}-5$ and substantial red shift is observed for $\mathrm{Cu}^{+} \mathrm{ZSM}-5$. The frequencies calculated for the extended model of copper active sites in ZSM-5 agree well with their experimental counterparts, which gives further validation of the model and supports the interpretation of the activation mechanism of $\mathrm{NO}$ over $\mathrm{Cu}^{+} \mathrm{ZSM}-5$ sketched in the preceding paragraph.

\section{Conclusions}

The proposed model of the copper active site in the MFI framework seems to be capable of describing wide scope of properties crucial for catalytic processes involved in DENOx on CuZSM-5. It should be stressed here that up to our knowledge this is the first realistic model of MFI framework which explains the source of the differences between $\mathrm{Cu}^{+}$and $\mathrm{Cu}^{2+}$ cations interacting with NO. At the same time it gives very physical insight into the mechanism of the activation of nitrogen oxide on $\mathrm{Cu}^{+} \mathrm{ZSM}-5$, which is known from its high catalytic activity ion DENOx. We hope to apply the tools presented here and confirmed by experiment, to get further valuable information on this catalytic process.

\section{Acknowledgements}

This study was sponsored by the grant of KBN 3 T09A 01017.

\section{References}

1. Iwamoto, M. Stud. Surf. Sci. Catal., 2000, 130, 23.

2. Kuroda, Y.; Maeda, H.; Yoshikawa, Y.; Kumashiro, R.; Nagao, M. J. Phys. Chem. B 1997, 101, 1312.

3. Hall, W. K. J. Phys. Chem. B, 1997. 101, 1979.

4. Polomino, G. T.; Fisicaro, P.; Bordiga, S.; Zecchina, A.; Giamello, E.; Lamberti, C. J. Phys. Chem. B, 2000. 104, 4064.

5. Sobalik, Z.; Tvaruzkova, Z.; Wichterlova, B. J. Phys. Chem.,2000, 102, 1077

6. Slinkin, A.; Kucherov, A.V. Catal. Today 1997, 36, 485; Kucherov, A. V.; Shigapov, A.N.; Ivanov, A.A.; Shelef, M. J. Catal, 1999, 186, 334.

7. Zecchina, A.; Bordiga, S.; Salvagio, M.; Spoto, G.; Scarano, D.; Lamberti, C. J. Catal., 1998, 173, 540. 
8. Beutel, T.; Sarkany, J.; Lei, G. D.; Jan, J. Y.; Sachtler, W. M. H. J. Phys. Chem., 1996, 100, 845.

9. Dedecek, J.; Wichtlerowa, B. Phys. Chem. Chem. Phys. 1999, 1, 629, and references therein

10. Wichterlova, B.; Dedecek, J.; Sobalik, Z.; Vondrova, A.; Klier, K. J. Catal. 1997,169, 194.

11. Lei, G. D.; Adejman, B.J.; Sarkany, J.; Sachtler, W.M.H. Appl. Catal. B, 1995, 5, 245.

12. Sartkany, J. J. Mol. Struct., 1997, 410-411, 145.

13. Sobalik, Z. Microp. Mesopor. Mater., 1998, 21, 525.

14. Sobalik, Z.; Tvaruzkova, Z.; Wichterlova, B. J. Phys. Chem., 1998, 102, 1077.

15. Broclawik, E.; Datka, J.; Gil, B.; Kozyra, P. Phys. Chem. Chem. Phys., 2000, 2, 401.

16. Broclawik, E.; Datka, J.; Gil, B.; Piskorz, W.; Kozyra, P. Topics in Catalysis, 2000, 11/12, 335.

17. Sayle, D.C.; Richard, C.; Catlow, A.; Gale, J. D.; Perrin, M. A.; Nortier, P. J. Phys. Chem. A, 1997, 101, 3331.

18. Nachtigallova, D.; Nachtigall, P.; Sierka, M.; Sauer, J. Phys. Chem. Chem. Phys. 1999, 1, 2019.

19. Rice, M. J.; Chakraborty, A. K.; Bell, A. T. J. Catal, 2000, 194, 278.

20. Nachtigall, P.; Nachtigallova, D.; Sauer, J. J. Phys. Chem. B 2000, 104, 1738.

21. Nachtigallova, D.; Nachtigall, P.; Sauer, J. Phys. Chem. Chem. Phys. 2001, 3, 1552.

22. DMol, Insight II release 96.0, User Guide, San Diego: Molecular Simulations, 1996.

23. Broclawik, E. In Modern Density Functional Theory: A Tool for Chemistry; Seminario, J.M. and Politzer, P., Eds; Elsevier: Amsterdam, 1995; p. 349.

24. Dedecek, J.; Sobalik, Z.; Tvaruzkova, Z.; Kaucky, D.; Wichterlova, B. J Phys. Chem. 1995, 99, 16327.

25. Wichterlova, B.; Dedecek, J.; Sobalik, Z. In Proc. $12^{\text {th }}$ Int. Zeolite Conf. In Baltimore; Treacy, M.M.J.; Marcus, B. K.; Bisher, M. E. and Higgins, J. B., Eds., MRS, 1999; p. 941.

26. Broclawik, E.; Datka, J.; Gil, B.; Kozyra, P. Topics in Catalysis, in print.

27. Broclawik, E.; Datka, J.; Gil, B. Kozyra, P. J. Phys. Chem, submitted.

(C) 2002 by MDPI (http://www.mdpi.org), Basel, Switzerland. 